\title{
Angiokeratoma circumscriptum neviforme: Extensive involvement of lower limb
}

\section{Tushar Kanti Sarkar¹, Nidhi Gupta², Debabrata Bandyopadhyay³, Shashank Bhargava²}

${ }^{1}$ Paikpari RH, Kolaghat, Purba Medinipur, WB, India, ${ }^{2} R$. D. Gardi Medical College, Ujjain, MP, India, ${ }^{3}$ Medical College

Kolkata, WB, India

Corresponding author: Dr. Shashank Bhargava, E-mail: shashank2811@gmail.com

\begin{abstract}
Angiokeratomas are a rare group of disorders characterized by ectasia of preexisting papillary dermal vessels. It presents itself with discrete and/or confluent hyperkeratotic plaques. Among the variants of angiokeratoma, angiokeratoma circumscriptum is the least common and its nevoid distribution is still rarer, typically present since birth or very early childhood. Herein, we report a case of multiple verrucous plaques on the right leg in a blaschkoid distribution extending up to the thigh in a 27-year-old male diagnosed as angiokeratoma circumscriptum neviforme (ACN) on clinical and histopathological grounds..
\end{abstract}

Key words: ACN; Blaschkoid; Verrucous

\section{INTRODUCTION}

Angiokeratomas are characterized by ectasia of superficial or papillary vessels with overlying hyperkeratosis [1], first described by Mibelli in 1889 and termed angiokeratoma circumscriptum by Dammert. The mechanism of development remains unknown [2]. Angiokeratomas may be localized or generalized. The localized forms have been classified into solitary angiokeratoma, angiokeratoma of Fordyce, angiokeratoma of Mibelli, and angiokeratoma circumscriptum neviforme (ACN). Among these, $\mathrm{ACN}$ is the rarest, characterized by the presence of hyperkeratotic papules developing into verrucous bluish-black plaques commonly on the lower limbs in a segmental distribution. A lack of spontaneous regression makes the elimination of ACN imperative by the use of an appropriate modality (diathermy, curettage, electrocautery, cryosurgery, deep excision followed by grafting, CO2/argon/KTP laser) [2,3].

\section{CASE REPORT}

A 27-year-old male presented himself to us with a history of multiple erythematous macules on the right leg distributed in a linear pattern, encroaching the thigh since childhood. Later, the lesions gradually progressed to form papules and plaques. Subsequently, the lesions became hyperkeratotic, then static. Although the hyperkeratotic lesions extended to the inner thigh, the scrotum was spared. A history of occasional pain and bleeding was present. There were no symptoms suggestive of systemic involvement. A family history was absent, neither was there a history of bleeding from other sites. An examination revealed the presence of multiple purplish verrucous hyperkeratotic plaques arranged in a blaschkoid fashion extending from the ankle to the inner thigh (Fig. la and b). The lesions were firm and tender, and no pulsation was felt. The rest of the leg was normal, and there was no asymmetry in the size of the legs. No bruit was heard on auscultation. The rest of the general and systemic examination was within normal limits. A complete hemogram and biochemistry were within normal limits. An X-ray revealed no bone or soft tissue abnormalities. An incisional biopsy for a histopathological examination revealed compact hyperkeratosis, acanthosis, and elongation of rete ridges. The papillary dermis showed the presence of multiple dilated, thin-walled, congested capillaries without extension to the deep dermis or subcutis (Fig. 2).

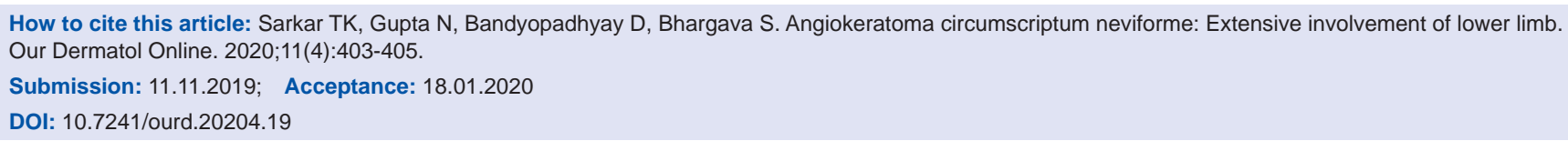




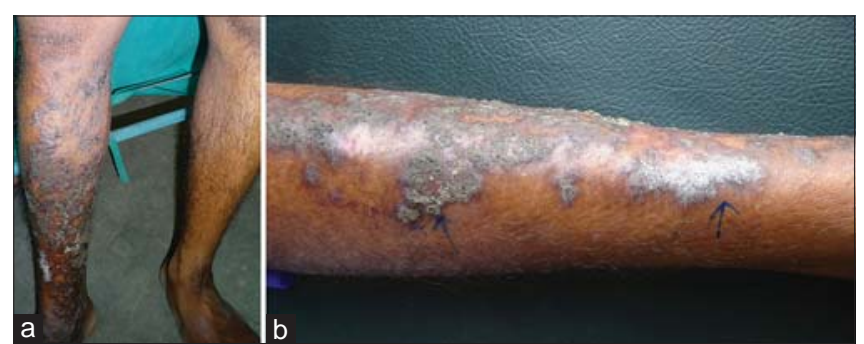

Figure 1: (a) Linear, verrucous, well-circumscribed plaques extending from the ankle to the inner thigh of the right side. (b) A close-up view of the linear verrucous lesions on the leg.

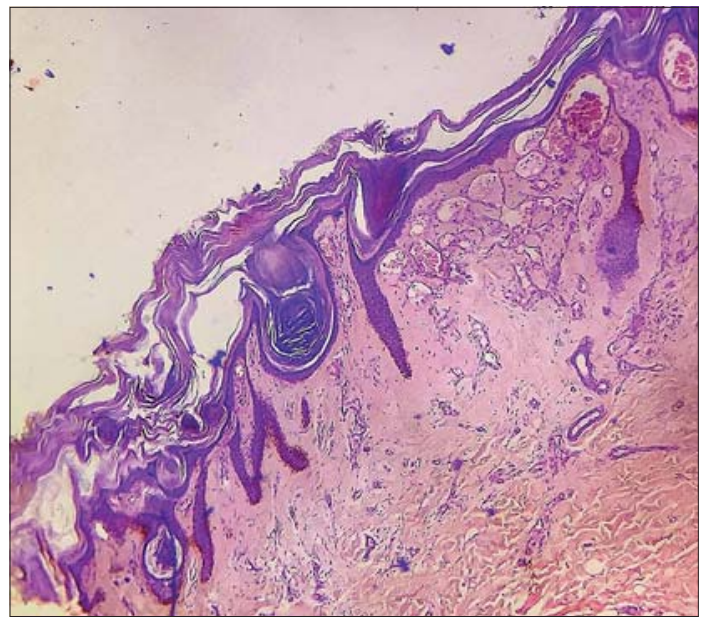

Figure 2: A photomicrograph showing compact hyperkeratosis, acanthosis, and elongated rete ridges, as well as the papillary dermis with dilated thin-walled vascular spaces containing erythrocytes (H\&E, 40X).

\section{DISCUSSION}

Angiokeratomas are telangiectasias of preexisting dermal capillaries and veins with hyperkeratotic surfaces [4]. It is three times more common in females than in males. Angiokeratoma is classified as localized or widespread. Angiokeratoma corporis diffusum is a systemic variety caused by a deficiency in any of several enzymes, resulting in the deposition of glycosphingolipids. Localized angiokeratomas are classified as angiokeratoma of Fordyce, located in the genital region, angiokeratoma of Mibelli, located on acral parts following exposure to cold, solitary and multiple angiokeratoma, and angiokeratoma circumscriptum, which is rare. Angiokeratoma circumscriptum neviforme is still a rarer variety of angiokeratoma, occurring at an early age. ACN is seen usually in infancy or early childhood, although lateonset cases have been reported [5]. ACN presents itself as bluish-black papules and nodules grouped to form a verrucous and hyperkeratotic plaque. Lesions are most commonly seen on the thighs and the gluteal region but may also occur on the neck [6]. Uncommon sites, such as the tongue, oral cavity, elbow, penis, and trunk, have also been reported [7-9]. Lesions are segmental or linearly arranged. The pathogenesis of ACN remains unknown but several causal factors, such as trauma, developmental anomaly, subcutaneous hematomas, pregnancy, and tissue asphyxia, have been suggested. Angiokeratoma circumscriptum have been reported to coexist with angiokeratoma of Fordyce and caviar spots (angiokeratoma of the tongue), Cobb syndrome, Klippel-Trénaunay syndrome, nevus flammeus, cavernous hemangioma, and traumatic arteriovenous fistula [3,10-12]. Clinically, angiokeratoma may mimic verrucous hemangioma, melanoma, pigmented basal cell carcinoma, and sometimes lichen planus hypertrophicus; in these cases, histopathology helps to differentiate between the conditions. HPE with ACN shows epidermal hyperkeratosis, papillomatosis, and/ or acanthosis, along with the presence of dilated blood vessels limited to the papillary dermis, unlike verrucous hemangioma, in which dilated capillaries extend deeper into the reticular dermis and subcutaneous tissue. In our case, correlating clinical features with the features of HPE confirmed the diagnosis of ACN. Treatment with diathermy, cryosurgery, curettage, and electrocautery is done for smaller lesions. Larger lesions require deep surgical excision or laser ablation with carbon dioxide [9], argon laser, or potassium titanyl phosphate (KTP) laser [2]. We planned carbon dioxide laser for this patient. Scanty case reports of this entity in the world literature prompted us to report this case.

\section{Consent}

The examination of the patient was conducted according to the principles of the Declaration of Helsinki.

The authors certify that they have obtained all appropriate patient consent forms, in which the patients gave their consent for images and other clinical information to be included in the journal. The patients understand that their names and initials will not be published and due effort will be made to conceal their identity, but that anonymity cannot be guaranteed.

\section{REFERENCES}

1. Sadana D, Sharma YK, Dash K, Chaudhari ND, Dharwadkar AA, Dogra BB. Angiokeratoma circumscriptum in a young male. Indian J Dermatol. 2014;59:85-7

2. Ari E, Çakmak SK, Çiftçi AY, Tamer E, Artüz F. A rare clinic type of angiokeratoma: angiokeratoma circumscriptum naeviforme. Our Dermatol Online. 2016;7:489-91.

3. Kassem Youssef H, Backobi E, Michel C. [Angiokeratoma circumscriptum neviforme]. Ann Dermatol Venereol. 2018;145:552-3.

4. Poddar I, Das A, Gharami RC. Angiokeratoma circumscriptum 
neviforme: Revisiting a rare entity. Indian J paediatr Dermatol. 2015;16:246-8.

5. Jindal SR, Chalvade P, Jerajani HR. Late onset palmar angiokeratoma circumscriptum: An unusual presentation. Indian Dermatol Online J. 2014;5:320-2.

6. Debbarman P, Roy S, Kumar P. Angiokeratoma circumscriptum neviforme. Indian Pediatr. 2012;49:80.

7. Aggarwal K, Jain VK, Jangra S, Wadhera R. Angiokeratoma circumscriptum of the tongue. Indian Pediatr. 2012;49:316-8.

8. Wang H, Zhang GY, Yu YQ. Variant distribution of skin lesions in angiokeratoma circumscriptum: Report of an untypical case. J Med Case. 2012;3:297-9.

9. Chaouche M, Cherif AD, El Kadiri S, Elloudi S, Baybay H, Mernissi FZ. A rare disease in childhood: angiokeratoma circumscriptum naviforme. Our Dermatol Online. 2019;10:e11.1-e11.3.

10. Das A, Mondal AK, Saha A, Chowdhury SN, Gharami RC.
Angiokeratoma circumscriptum neviforme: An entity, few and far between. Indian Dermatol Online J. 2014;5:472-4.

11. Wankhade V, Singh R, Sadhwani V, Kodate P, Disawal A. Angiokeratoma circumscriptum naeviforme with soft tissue hypertrophy and deep venous malformation: A variant of KlippelTrenaunay syndrome? Indian Dermatol Online J. 2014;5(Suppl 2):S109-12

12. Samudrala S, Bhat MR. Recurrent, nodular lesions over the vulva: A diagnostic challenge. Indian J Sex Transm Dis AIDS. 2018;39:124-6.

Copyright by Tushar Kanti Sarkar, et al. This is an open-access article distributed under the terms of the Creative Commons Attribution License, which permits unrestricted use, distribution, and reproduction in any medium, provided the original author and source are credited.-

Source of Support: Nil, Conflict of Interest: None declared. 